\title{
Parâmetros relevantes na determinação da velocidade terminal de frutos de café ${ }^{1}$
}

\author{
Sandra M. Couto², Anderson C. Magalhães ${ }^{3}$, Daniel M. de Queiroz ${ }^{4}$ \& Itaciane T. Bastos ${ }^{5}$ \\ 1 Trabalho de pesquisa desenvolvido com recursos financeiros do Consórcio Brasileiro de Pesquisa e Desenvolvimento \\ em Café - CBP\&D Café \\ 2 DEA/UFV. Av. Ph Rolfs s/n. CEP 36571-000, Viçosa, MG. Fone: (31) 3899 1925, E-mail: scouto@mail.ufv.br (Foto) \\ ${ }^{3}$ DEA/UFV. Fone: (31) 3899 2729, E-mail: achagas@alunos.ufv.br \\ 4 DEA/UFV. Fone: (31) 3899 1882, E-mail: queiroz@mail.ufv.br \\ ${ }^{5}$ DEA/UFV
}

Protocolo $147-24 / 10 / 2001$ - Aprovado em 31/1/2003

\begin{abstract}
Resumo: No desenvolvimento de máquinas de separação movidas a fluxos de ar, o conhecimento da velocidade terminal de um produto e das impurezas associadas é de importância fundamental. Neste trabalho, investigou-se o comportamento dos valores da velocidade terminal de frutos de café (variedades Catuaí e Híbrido Timor) em função dos parâmetros (a) estádio de maturação do produto na colheita ("verde" e "cereja"); (b) época de colheita; (c) variedade; (d) teor de umidade dos frutos e (e) número de frutos contidos na amostra usada para as determinações da velocidade. A análise dos resultados mostrou que os valores das velocidades terminais foram influenciados, significativamente, pelo teor de umidade do produto, estádio de maturação dos frutos e tamanho das amostras usadas nas determinações; entretanto, a época de colheita e a variedade dos frutos não se mostraram como parâmetros importantes nas determinações da velocidade terminal do produto. Relações funcionais entre parâmetros adimensionais, usando-se análise dimensional e o teorema Pi de Buckingham, foram também obtidas e são apresentadas neste trabalho; por outro lado, essas relações fornecem estimativas razoáveis para a velocidade terminal de um fruto, em função das velocidades de flutuação determinadas, experimentalmente, para amostras com um número maior de frutos.
\end{abstract}

Palavras-chave: Coffea arábica L., velocidade terminal, teor de umidade, estádio de maturação

\section{Important parameters for determination of terminal velocity of coffee fruits}

\begin{abstract}
The knowledge of the terminal velocity of a product is of fundamental importance in the development of separation machines using airflows. The behavior of the values of the terminal velocity of coffee fruits (varieties Catuaí and Hybrid Timor) was investigated in this work as a function of the following parameters: (a) maturation level of the coffee at the harvesting ("green" and "cherry" fruits); (b) harvesting time; (c) variety; (d) moisture content of the fruits and (e) number of fruits in the sample used for the determinations of the terminal velocity. Analysis of the results showed that the values of the terminal velocity were significantly influenced by the moisture content of product, fruit maturation level and size of the samples used in the determinations. However, the harvesting time and the fruit variety did not present as important parameters in the terminal velocity determinations. Functional relationships among dimensionless parameters, using dimensional analysis and the Buckingham $\mathrm{Pi}$ theorem were also obtained and presented in this work. These relationships may be used to estimate the terminal velocity of one fruit when experimental data from the fluctuation velocity of samples with a larger number of fruits are known.
\end{abstract}

Key words: Coffea arábica L., terminal velocity, moisture content, maturation level

\section{INTRODUÇÃO}

O beneficiamento de um produto é essencial à tecnologia de produção de sementes de alta qualidade e dele dependem os produtores para colocarem seu produto em condições competitivas de comercialização. A qualidade do produto beneficiado resulta da capacidade das máquinas em remover impurezas, sementes de má qualidade e todo material estranho.
A aplicação de fluxo de ar para a separação de um produto de materiais indesejados tem sido usada por um grande número de pesquisadores. Srivastava et al. (1993), analisando sistemas de separação utilizados em máquinas colhedoras, verificaram a necessidade de desenvolvimento de um separador eficiente, compacto e independente da força de gravidade .

Para o desenvolvimento de máquinas em que se utiliza um fluxo de ar para separar os grãos das impurezas, o conhecimento 
da velocidade terminal de todas as partículas que compõem uma mistura de produto e impurezas, tais como palha, sementes silvestres e folhas, é essencial (Mohsenin, 1978).

Reed et al., citados por Teixeira (1995) investigaram os parâmetros que mais influenciavam na separação de misturas de grãos e impurezas e concluíram que a eficiência de separação, ao serem utilizadas peneiras, está relacionada à taxa de alimentação do produto e ao seu teor de impureza inicial.

Uhl \& Lamp (1966) investigaram o efeito da velocidade do ar na separação das impurezas contidas em amostras de trigo, milho e soja. As faixas de velocidade do ar determinadas para a separação pneumática foram $5,79-9,14 ; 7,92-12,80$ e $9,14-18,29 \mathrm{~m} \mathrm{~s}^{-1}$ para milho, trigo e soja, respectivamente. Ao utilizarem um único fluxo de ar no processo de separação das impurezas, verificaram a ocorrência de uma separação completa para o trigo e a soja, mas para o milho ocorreram enormes perdas de grão.

Segundo Srivastava et al. (1993), o tamanho e a massa dos grãos e impurezas são as propriedades mais importantes na limpeza, sendo o fluxo de ar intimamente influenciado pela massa. Existem, também, outros fatores que influenciam no movimento relativo entre sólido-fluido, dentre os quais a orientação do sólido durante o movimento e a rugosidade do produto.

Segundo Mohsenin (1978) estudos aerodinâmicos de grãos agrícolas têm mostrado que esses assumem, a todo instante, orientações aleatórias, giram em torno do eixo vertical e, ainda, que existe tendência de sua maior dimensão movimentar-se na direção do plano horizontal. Esta rotação e inclinação têm sido explicadas como resultado da falta de simetria do grão e, neste caso, a força aerodinâmica que atua no corpo não passa pelo seu centro de gravidade e a sua direção não é paralela à da velocidade do ar. Esta força pode ser considerada equivalente à soma de uma força vertical passando pelo centro de gravidade, uma força horizontal que tende a girar o grão em torno do eixo vertical, e um binário, no plano vertical, que tende a mudar a inclinação do eixo mais longo do grão. Observa-se, experimentalmente que, quando os grãos começam a girar, eles começam, também, a subir em um túnel de vento, indo para uma região de menor velocidade; em outras palavras, a rotação causa maior arraste e menor velocidade terminal.

$\mathrm{O}$ acréscimo de elementos de rugosidade à superfície de um corpo pode suprimir oscilações locais na transição entre o escoamento laminar e o turbulento na camada limite. Essas oscilações podem conduzir a variações de arrasto e a flutuações aleatórias da sustentação (Fox \& McDonald, 1998).

A determinação experimental da velocidade terminal de um corpo é, freqüentemente, realizada de duas maneiras: (a) determinando a velocidade necessária para que o corpo flutue em um fluxo de ar ascendente (Carman, 1996; Singh \& Goswami, 1996; Allen \&Watts, 1997; Gupta \& Das, 1997; Tabak \& Wolf, 1998; Nimkar \& Chattopadhyay, 2001, entre outros) e (b) por meio da medição do deslocamento, em função do tempo, de uma partícula em queda livre no ar, cuja equação de movimento é:

$$
\mathrm{m} \frac{\mathrm{d}^{2} \mathrm{x}}{\mathrm{dt}^{2}}=\mathrm{mg}-\mathrm{F}_{\mathrm{r}}
$$

em que:

$$
\begin{array}{ll}
\mathrm{m} & \text { - massa do corpo, } \mathrm{kg} \\
\mathrm{X} & \text { - deslocamento da partícula, } \mathrm{m} \\
\mathrm{g} & \text { - aceleração da gravidade, } \mathrm{m} \mathrm{s}^{-2} \\
\mathrm{~F}_{\mathrm{r}} & \text { - força resistente, } \mathrm{N}
\end{array}
$$

A solução da Eq. (1), quando o corpo atinge a velocidade terminal, $\mathrm{V}_{\mathrm{t}}$, é:

$$
\mathrm{x}=\frac{\mathrm{V}_{\mathrm{t}}}{\mathrm{g}} \ln \left[\cosh \left(\frac{\mathrm{g}}{\mathrm{V}_{\mathrm{t}}} \mathrm{t}\right)\right]
$$

Os fatores que afetam os valores da velocidade terminal de frutos de café não são, ainda, bem conhecidos, e podem ser diferentes daqueles que influenciam outros tipos de grão. Ressalta-se, por exemplo, que o comportamento da rugosidade da superfície de frutos de café difere visivelmente daquele para outros grãos agrícolas. À medida que o teor de umidade deste produto é reduzido, a rugosidade superficial aumenta.

No desenvolvimento de máquinas de separação que utilizam um fluxo de ar, o conhecimento da velocidade terminal de um produto e das impurezas associadas é de importância fundamental. Assim, desenvolveu-se este trabalho com o objetivo de determinar parâmetros que influenciam os valores da velocidade terminal de café. Especificamente, foram investigados os seguintes parâmetros: (a) estádio de maturação do café na época da colheita; (b) época de colheita; (c) variedade; (d) teor de umidade dos frutos e (e) número de frutos na amostra.

\section{MATERIAL E MÉTODOS}

Frutos de café das variedades Catuaí e Híbrido Timor colhidos no sistema convencional na forma de derriça no pano, de uma plantação localizada a uma latitude de $20^{\circ} 52^{\prime} 04^{\prime \prime} \mathrm{S}$, com altitude de $657 \mathrm{~m}$, em uma região de Viçosa, $\mathrm{MG}$, foram separados nas categorias "verde" e "cereja". O café da variedade Catuaí foi colhido em duas épocas diferentes e é diferenciado no texto, pela nomenclatura lotes 1 e 2 .

Para a obtenção do produto com cinco teores de umidade diferentes, frutos de café das duas variedades foram secados em terreiro de cimento. Os teores de umidade dos frutos foram determinados, em todas as etapas do experimento, pelo método da estufa a $105^{\circ} \mathrm{C}$ por $24 \mathrm{~h}$.

Para a determinação da velocidade terminal dos grãos de café, utilizou-se um dispositivo constituído de um ventilador centrífugo acoplado a um tubo de acrílico transparente $(0,093 \mathrm{~m}$ de diâmetro interno e 2,0 m de comprimento) como esquematizado na Figura 1. Por outro lado, a amostra de frutos de café, depois de ser acomodada em um compartimento telado posicionado a 1,0 $\mathrm{m}$ da extremidade inferior do tubo de acrílico, foi submetida a um fluxo de ar crescente, até que ocorresse o início de flutuação dos grãos. A velocidade do ar, nesta condição, era determinada por meio de um anemômetro e considerada a velocidade terminal do produto. 


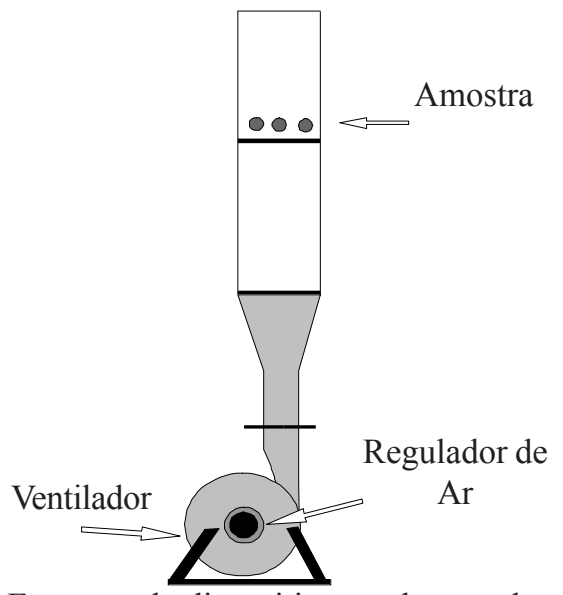

Figura 1. Esquema do dispositivo usado para determinação da velocidade terminal

Para se investigar os efeitos do estádio de maturação do café na colheita, da época de colheita, da variedade e do teor de umidade dos frutos nos valores das velocidades terminais, trabalhou-se com frutos das variedades Catuaí (lotes 1 e 2) e Timor, obtendo-se as velocidades necessárias para a flutuação de um único fruto proveniente de cada combinação "variedade - teor de umidade - grau de maturação". Em cada situação foram realizadas três repetições.

$\mathrm{Na}$ investigação do efeito do número de frutos a serem postos a flutuar simultaneamente nos valores das velocidades terminais, trabalhou-se com amostras contendo 1, 5 e 10 grãos de café das variedades Catuaí (lote 2) e Timor classificados, depois da colheita, nas categorias "verde" e "cereja". As determinações foram realizadas em três repetições, usando-se sempre frutos diferentes.

\section{RESULTADOS E DISCUSSÃO}

Na Tabela 1 tem-se valores das velocidades terminais, acompanhados dos respectivos desvios-padrão, de amostras contendo um único fruto de café, das variedades Catuaí (lotes 1 e 2) e Timor, a diferentes teores de umidade e graus de maturação. Cada valor representa a média de três determinações.

Observa-se, ainda na Tabela 1 , que os valores das velocidades terminais, que variaram de 10,2 a $16,8 \mathrm{~m} \mathrm{~s}^{-1}$, tendem a reduzir com a diminuição da umidade no produto e a serem maiores para os frutos "cereja". Nota-se, também, que os desvios-padrão envolvidos nas determinações desta grandeza para frutos da variedade Catuaí, lote 1, foram os maiores, caso em que os desvios apresentados pelos valores das velocidades terminais dos frutos "verde" superam os dos "cereja".

\section{Efeito do teor de umidade}

As equações polinomiais, de até segundo grau, ajustadas (regressão) aos dados experimentais para expressar o comportamento da velocidade terminal dos grãos, em função do teor de umidade, são apresentadas na Figura 2. Todos os coeficientes foram significativos ao nível de $5 \%$ de probabilidade. Observase que a influência do teor de umidade foi significativa nos valores das velocidades terminais em todos os casos investigados e que, para a maioria das combinações "variedade - grau
Tabela 1. Valores médios da velocidade terminal ( $\left.\mathrm{M}, \mathrm{m} \mathrm{s}^{-1}\right)$, com os respectivos desvios-padrão (DP, $\mathrm{m} \mathrm{s}^{-1}$ ) de um único grão de café a diferentes teores de umidade (TU, \% b.u.)

\begin{tabular}{|c|c|c|c|c|c|c|c|c|}
\hline \multicolumn{9}{|c|}{ Variedade } \\
\hline \multicolumn{6}{|c|}{ Catuaí } & \multicolumn{3}{|c|}{ Timor } \\
\hline \multicolumn{3}{|c|}{ Lote 1} & \multicolumn{3}{|c|}{ Lote 2} & \multirow[b]{2}{*}{$\mathrm{TU}$} & \multirow[b]{2}{*}{ M } & \multirow[b]{2}{*}{ DP } \\
\hline TU & M & $\mathrm{DP}^{*}$ & TU & M & DP & & & \\
\hline \multicolumn{9}{|c|}{ Cereja } \\
\hline 67,5 & 16,0 & 0,4 & 70,4 & 16,8 & 0,1 & 62,6 & 16,20 & 0,10 \\
\hline 61,4 & 14,4 & 0,1 & 57,8 & 15,2 & 0,2 & 53,0 & 14,30 & 0,10 \\
\hline 46,6 & 14,4 & 0,6 & 40,4 & 14,1 & 0,1 & 40,1 & 13,20 & 0,20 \\
\hline 29,3 & 13,4 & 0,7 & 28,8 & 13,1 & 0,1 & 22,9 & 12,60 & 0,20 \\
\hline 18,7 & 12,3 & 0,5 & 13,3 & 11,1 & 0,2 & 11,0 & 11,70 & 0,30 \\
\hline \multicolumn{9}{|c|}{ Verde } \\
\hline 67,9 & 15,7 & 1,1 & 68,0 & 15,5 & 0,2 & 65,2 & 15,20 & 0,10 \\
\hline 61,4 & 14,4 & 0,9 & 61,0 & 14,7 & 0,3 & 55,1 & 14,10 & 0,10 \\
\hline 51,1 & 14,1 & 0,6 & 50,7 & 13,8 & 0,2 & 41,5 & 12,27 & 0,04 \\
\hline 30,3 & 12,0 & 1,3 & 35,9 & 12,1 & 0,2 & 18,5 & 11,20 & 0,20 \\
\hline 17,2 & 11,5 & 0,2 & 12,6 & 12,0 & 0,1 & 19,5 & 10,20 & 0,20 \\
\hline
\end{tabular}

de maturação", as velocidades terminais dos frutos variaram linearmente com os seus teores de umidade; apenas no caso de frutos das variedades Timor "cereja" e Catuaí "verde", lote 2 , essas variações foram quadráticas.

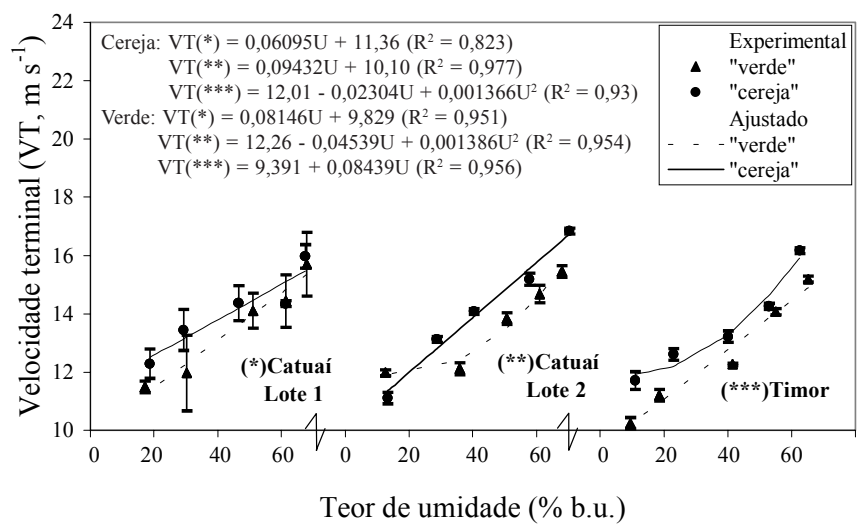

Figura 2. Velocidade terminal de um único grão de café, em função do teor de umidade

A Figura 2 exibe as curvas provenientes das equações ajustadas, juntamente com os valores médios experimentais e intervalos de variação, construídos com base no desvio-padrão.

\section{Efeito do estádio de maturação na colheita}

Ao se utilizar as equações ajustadas em um intervalo de umidade comum a todas as variedades e graus de maturação do produto na colheita (de 18,7 a $62,6 \%$ b.u.) verifica-se que as velocidades terminais dos grãos "cereja” são, em média, 5,5, 7,6 e 7,7\% superiores às dos grãos "verde" para as variedades Catuaí, lotes 1 e 2, e Timor, respectivamente. Vê-se, assim, que o grau de maturação dos frutos na colheita exerce influência nos valores das velocidades terminais. Entretanto, para frutos Catuaí do lote 1, as diferenças entre os valores das velocidades terminais de frutos "cereja" e "verde", se encontram na mesma faixa de valores dos desvios experimentais.

\section{Efeito da época de colheita}

A contribuição da época de colheita dos frutos da variedade Catuaí nas variações dos valores da velocidade terminal é muito 
pequena em relação àquela, devido ao grau de maturação; as velocidades dos frutos do lote 1 , quando comparadas com as do lote 2 , são $0,4 \%$ inferiores para grãos "cereja", e 1,5\% superiores, para frutos "verde".

\section{Efeito da variedade do fruto de café}

Quando se considera o produto "cereja", as velocidades terminais dos frutos da variedade Catuaí, lotes 1 e 2, são, respectivamente, 2,3 e $2,7 \%$ maiores que as do produto da variedade Timor. Para o produto no estádio de maturação "verde", as velocidades dos frutos da variedade Catuaí, lotes 1 e 2, são, respectivamente, 4,4 e 2,9\% maiores que as do produto da variedade Timor. Os desvios devidos à variedade encontramse na mesma faixa dos experimentais mostrando, assim, que este parâmetro não parece influenciar significativamente na velocidade terminal do produto.

Para o intervalo comum de umidade e ao se desconsiderar a variedade do grão e o seu grau de maturação, observa-se que os valores dos desvios médios envolvidos na velocidade terminal resultante variam de 0,53 a $0,59 \mathrm{~m} \mathrm{~s}^{-1}$, com valor médio de $0,57 \mathrm{~m} \mathrm{~s}^{-1}$. Esses desvios se encontram na faixa de valores dos desvios experimentais obtidos para as velocidades terminais dos grãos da variedade Catuaí, lote 1, porém são superiores àqueles encontrados para as velocidades dos grãos das variedades Catuaí, lote 2, e Timor. Sob essas condições, a velocidade terminal média varia entre 11,8 e $15,3 \mathrm{~m} \mathrm{~s}^{-1}$; se, além dessas considerações, o efeito do teor de umidade for desprezado, obter-se-á o valor de $13,3 \mathrm{~m} \mathrm{~s}^{-1}$ para a velocidade terminal média dos frutos e, neste caso, o desvio se torna igual a $1,2 \mathrm{~m} \mathrm{~s}^{-1}$, que se encontra também na faixa de valores determinada para a velocidade terminal de grãos Catuaí, lote 1 .

Portanto, verifica-se efeito significativo do teor de umidade, variedade e estádio de maturação na época de colheita nos valores das velocidades terminais de frutos Catuaí, lote 2, e Timor. Os grandes desvios apresentados pelas velocidades terminais dos frutos Catuaí, lote 1, impossibilitaram, no caso, a determinação da influência desses parâmetros.

\section{Efeito do tamanho da amostra de frutos}

A Tabela 2 apresenta os valores das velocidades terminais obtidas experimentalmente para amostras de café das variedades Catuaí (lote 2) e Timor, contendo 1, 5 e 10 frutos a diferentes teores de umidade e graus de maturação. Nota-se que para cada tamanho de amostra, as velocidades terminais dos frutos "cereja" são maiores que as dos "verde". Observase, a medida em que se aumenta o número de frutos nas amostras, que a velocidade terminal média de três repetições tende a diminuir, o que acontece para as duas variedades, nos diferentes graus de maturação e de teor de umidade.

A análise de variância realizada nos dados experimentais, provenientes de cada combinação "variedade-grau de maturação", mostra que os valores da velocidade terminal do produto foram afetados significativamente, ao nível de $5 \%$ de probabilidade, pelo teor de umidade e pelo tamanho da amostra, sendo a interação desses dois fatores, também, significativa. $\mathrm{O}$ efeito do tamanho da amostra nos valores das velocidades terminais para cada combinação "variedade-grau de maturaçãoteor de umidade", foi investigado por meio da aplicação de testes de comparação de médias (Newman Keuls, ao nível de $5 \%$ de probabilidade). Os resultados mostram que as velocidades terminais de amostras contendo 1, 5 e 10 frutos, diferem significativamente.

Partículas múltiplas atuam como obstrução para o escoamento de ar, de maneira similar a uma peneira colocada perpendicularmente ao escoamento. Assim, ao se reduzir o número de partículas, a turbulência da correnteza livre tenderá a diminuir e isto faz com que o coeficiente de arrasto diminua. Ressalta-se que as velocidades terminais apresentadas na Tabela 2 são as velocidades do ar na correnteza livre, medidas em uma seção transversal do duto abaixo do local de flutuação dos frutos. Sob condições de escoamento de ar em regime permanente, a obstrução da área de escoamento pelos frutos de café faz com que estes fiquem submetidos a uma velocidade do ar maior que aquela medida na correnteza livre. Se as velocidades terminais das amostras contendo 5 e 10 frutos

Tabela 2. Valores médios das velocidades terminais $\left(\mathrm{M}, \mathrm{m} \mathrm{s}^{-1}\right)$ de amostras de café, variedades Timor e Catuaí lote 2, com diferentes números de frutos e respectivos desvios-padrão (d.p.)

\begin{tabular}{|c|c|c|c|c|c|c|c|c|c|c|c|c|c|}
\hline \multirow{4}{*}{$\begin{array}{l}\text { Teor de } \\
\text { Umidade } \\
\text { (\% b.u.) }\end{array}$} & \multicolumn{6}{|c|}{ Catuaí (Lote 2) } & \multirow{4}{*}{$\begin{array}{l}\text { Teor de } \\
\text { Umidade } \\
\text { (\% b.u.) }\end{array}$} & \multicolumn{6}{|c|}{ Timor } \\
\hline & \multicolumn{6}{|c|}{ Número de Grãos } & & \multicolumn{6}{|c|}{ Número de Grãos } \\
\hline & \multicolumn{2}{|c|}{1} & \multicolumn{2}{|c|}{5} & \multicolumn{2}{|c|}{10} & & \multicolumn{2}{|c|}{1} & \multicolumn{2}{|c|}{5} & \multicolumn{2}{|c|}{10} \\
\hline & M & d.p. & M & d.p. & M & d.p. & & M & d.p. & M & d.p. & $\mathrm{M}$ & d.p. \\
\hline \multicolumn{14}{|c|}{ Cereja } \\
\hline 70,4 & 16,84 & 0,10 & 15,49 & 0,45 & 14,59 & 0,14 & 62,6 & 16,17 & 0,14 & 14,51 & 0,28 & 13,93 & 0,08 \\
\hline 57,8 & 15,19 & 0,17 & 14,26 & 0,10 & 13,70 & 0,07 & 53,0 & 14,26 & 0,10 & 13,64 & 0,18 & 12,29 & 0,06 \\
\hline 40,4 & 14,08 & 0,06 & 13,29 & 0,13 & 12,42 & 0,12 & 40,1 & 13,22 & 0,20 & 11,97 & 0,06 & 10,36 & 0,27 \\
\hline 28,8 & 13,13 & 0,06 & 12,18 & 0,08 & 11,09 & 0,10 & 22,9 & 12,61 & 0,22 & 10,98 & 0,22 & 9,95 & 0,14 \\
\hline 13,3 & 11,11 & 0,23 & 10,38 & 0,17 & 9,75 & 0,13 & 11,0 & 11,71 & 0,33 & 10,16 & 0,11 & 9,53 & 0,26 \\
\hline Média & 14,07 & 0,12 & 13,12 & 0,19 & 12,31 & 0,11 & Média & 13,59 & 0,20 & 12,25 & 0,17 & 11,21 & 0,16 \\
\hline d.p. & 2,16 & & 1,96 & & 1,95 & & d.p. & 1,71 & & 1,81 & & 1,85 & \\
\hline \multicolumn{14}{|c|}{ Verde } \\
\hline 68,0 & 15,46 & 0,20 & 14,73 & 0,16 & 13,95 & 0,03 & 65,2 & 15,19 & 0,08 & 14,32 & 0,07 & 13,34 & 0,23 \\
\hline 61,0 & 14,68 & 0,29 & 13,90 & 0,19 & 13,08 & 0,09 & 55,1 & 14,07 & 0,08 & 12,57 & 0,17 & 11,87 & 0,15 \\
\hline 50,7 & 13,84 & 0,15 & 12,98 & 0,19 & 11,99 & 0,11 & 41,5 & 12,27 & 0,04 & 11,51 & 0,17 & 10,61 & 0,12 \\
\hline 35,9 & 12,11 & 0,22 & 11,16 & 0,11 & 10,99 & 0,19 & 18,5 & 11,21 & 0,18 & 10,16 & 0,16 & 9,67 & 0,19 \\
\hline 12,6 & 11,98 & 0,10 & 10,11 & 0,20 & 9,70 & 0,16 & 9,5 & 10,24 & 0,19 & 9,33 & 0,06 & 9,08 & 0,07 \\
\hline Média & 13,61 & 0,19 & 12,57 & 0,17 & 11,94 & 0,12 & Média & 12,60 & 0,12 & 11,58 & 0,12 & 10,91 & 0,15 \\
\hline d.p. & 1,54 & & 1,91 & & 1,68 & & d.p. & 2,03 & & 1,97 & & 1,72 & \\
\hline
\end{tabular}


forem comparadas com aquelas para 1 fruto, consideradas mais próximas das verdadeiras, verifica-se que os maiores desvios ocorrem quando dez frutos são colocados a flutuar (Tabela 2).

Para se investigar a existência de um relacionamento entre os valores das velocidades terminais, determinados para amostras contendo diferentes números de frutos, empregouse a técnica da análise dimensional, visando-se obter relações funcionais entre parâmetros adimensionais. Esses parâmetros foram obtidos por meio do teorema Pi de Buckingham (Fox \& McDonald, 1998). A velocidade terminal $\left(\mathrm{V}_{\mathrm{NG}}\right)$ de uma amostra contendo $\mathrm{N}$ frutos, parâmetro dependente, foi considerada função dos seguintes parâmetros independentes: $V_{1}$ (velocidade terminal de um fruto); $A_{D}$ (área da seção transversal do duto); $\mathrm{W}_{\mathrm{TP}}$ (peso total de frutos na seção transversal do duto); $\rho_{\mathrm{p}}$ (massa específica real do fruto) e $\mathrm{D}_{\mathrm{P}}$ (diâmetro médio do fruto). Dois grupos de variáveis independentes foram considerados:

$$
\begin{aligned}
& \text { Caso (1): } \mathrm{V}_{\mathrm{NG}}=\mathrm{f}\left(\mathrm{V}_{1}, \mathrm{~A}_{\mathrm{D}}, \mathrm{W}_{\mathrm{TP}}, \mathrm{D}_{\mathrm{PT}}\right) \mathrm{e} \\
& \text { Caso (2): } \mathrm{V}_{\mathrm{NG}}=\mathrm{f}\left(\mathrm{V}_{1}, \mathrm{~A}_{\mathrm{D}}, \rho_{\mathrm{P}}, \mathrm{W}_{\mathrm{TP}}, \mathrm{D}_{\mathrm{P}}\right)
\end{aligned}
$$

Para a aplicação do teorema Pi de Buckingham, as três primeiras variáveis independentes das Eqs. (3) e (4) foram fixadas e obtidas as seguintes dependências:

- para o caso (1):

$$
\frac{\mathrm{V}_{\mathrm{NG}}}{\mathrm{V}_{1}}=\mathrm{f}_{1}\left(\frac{\mathrm{D}_{\mathrm{P}}}{\mathrm{A}_{\mathrm{D}}^{1 / 2}}\right)
$$

e

- para o caso (2):

$$
\frac{V_{N G}}{V_{1}}=f_{2}\left(\frac{D_{P}}{A_{D}^{1 / 2}}, \frac{W_{T P}}{V_{1}^{2} A_{D} \rho_{P}}\right)=f_{2}([A],[B])
$$

A forma dos funcionais $\mathrm{f}_{1}$ e $\mathrm{f}_{2}$ foram obtidas por meio de regressão linear aplicada aos dados experimentais. O primeiro caso, considerando-se $[\mathrm{A}]=\left[\left(\mathrm{N} \mathrm{D}_{\mathrm{P}}\right) /\left(\mathrm{A}_{\mathrm{D}}{ }^{1 / 2}\right)\right]$ é referenciado no texto como Método 1, enquanto no segundo caso foram realizadas as seguintes variações:

- Método A:

$$
[A]=\left(\frac{N D_{P}}{A_{D}^{1 / 2}}\right)
$$

e

$$
[B]=\frac{\mathrm{gNm}_{\mathrm{P}}}{\mathrm{V}_{1}^{2} \mathrm{~A}_{\mathrm{D}} \rho_{\mathrm{P}}} \quad\left(\mathrm{em} \text { que }: \mathrm{W}_{\mathrm{TP}}=\mathrm{gNm}_{\mathrm{p}}\right)
$$

- Método B:

$$
[\mathrm{A}]=\left(\frac{\mathrm{D}_{\mathrm{P}}}{\mathrm{A}_{\mathrm{D}}^{1 / 2}}\right)
$$

e

$$
[\mathrm{B}]=\frac{\mathrm{gNm}_{\mathrm{P}}}{\mathrm{V}_{1}^{2} \mathrm{~A}_{\mathrm{D}} \rho_{\mathrm{P}}} \quad\left(\mathrm{em} \text { que }: \mathrm{W}_{\mathrm{TP}}=\mathrm{gNm}_{\mathrm{p}}\right)
$$

- Método C:

$$
[\mathrm{A}]=\left(\frac{\mathrm{N} \mathrm{D}}{\mathrm{A}_{\mathrm{D}}^{1 / 2}}\right)
$$

e

$$
[\mathrm{B}]=\frac{\mathrm{gNv}_{\mathrm{P}}}{\mathrm{V}_{1}^{2} \mathrm{~A}_{\mathrm{D}}}
$$

e

- Método D:

$$
[\mathrm{A}]=\left(\frac{\mathrm{D}_{\mathrm{P}}}{\mathrm{A}_{\mathrm{D}}^{1 / 2}}\right)
$$

$$
[\mathrm{B}]=\frac{\mathrm{gNv}_{\mathrm{P}}}{\mathrm{V}_{1}^{2} \mathrm{~A}_{\mathrm{D}}}
$$

em que:

$$
\begin{array}{ll}
\mathrm{m}_{\mathrm{P}} & \text { - massa unitária do fruto, } \mathrm{kg} \\
\mathrm{v}_{\mathrm{P}} & \text { - volume unitário do fruto, } \mathrm{m}^{3} \\
\mathrm{~g} & \text { - aceleração da gravidade, } \mathrm{m} \mathrm{s}^{-2}
\end{array}
$$

Usando-se as relações funcionais obtidas para cada um dos métodos e os dados experimentais para frutos "cereja" da variedade Catuaí, as velocidades terminais de amostras contendo 1 fruto foram estimadas a partir das outras, contendo 5 e 10 frutos. A Tabela 3 apresenta os desvios, em relação à velocidade terminal medida para um fruto, ao se estimar esta velocidade usando-se os resultados experimentais para cinco, $\mathrm{V}_{1}(5)$ e dez frutos, $\mathrm{V}_{1}(10)$. Esses desvios podem, também, ser visualizados na Figura 3.

Observa-se, na Tabela 3 e Figura 3, que os maiores desvios ocorreram ao se estimar a velocidade terminal de 1 grão a partir daquela medida para 10 frutos. Neste caso, considerando-se todos os teores de umidade, o Método 1 foi o que proporcionou o menor desvio médio na estimativa da velocidade de um fruto. 
Tabela 3. Desvios, em relação à velocidade terminal medida para um grão, ao se estimar a velocidade para um fruto, usando-se os resultados experimentais para cinco $\mathrm{V}_{1}(5)$ e dez frutos $\mathrm{V}_{1}(10)$ da variedade Catuaí, na condição "cereja"

\begin{tabular}{lllccccc}
\hline & & \multicolumn{6}{c}{ Teor de Umidade (\% b.u.) } \\
\cline { 3 - 8 } & & 70,4 & 57,8 & 40,4 & 28,8 & 13,3 & Média \\
\hline Método 1 & v1(5) & 0,019 & 0,145 & 0,115 & 0,198 & 0,004 & 0,096 \\
& v1(10) & 0,022 & 0,347 & 0,018 & 0,518 & 0,035 & 0,188 \\
Método A & v1(5) & 0,186 & 0,003 & 0,041 & 0,159 & 0,025 & 0,083 \\
& v1(10) & 0,091 & 0,417 & 0,063 & 0,369 & 0,102 & 0,208 \\
Método B & v1(5) & 0,250 & 0,009 & 0,088 & 0,139 & 0,052 & 0,107 \\
& v1(10) & 0,077 & 0,368 & 0,178 & 0,341 & 0,261 & 0,245 \\
Método C & v1(5) & 0,190 & 0,020 & 0,045 & 0,151 & 0,008 & 0,083 \\
& v1(10) & 0,084 & 0,368 & 0,070 & 0,351 & 0,141 & 0,203 \\
Método D & v1(5) & 0,230 & 0,045 & 0,085 & 0,134 & 0,058 & 0,110 \\
& v1(10) & 0,039 & 0,290 & 0,166 & 0,324 & 0,280 & 0,220 \\
\hline
\end{tabular}

Os resultados das análises de regressão mostraram que os coeficientes relacionados ao parâmetro [B], nos métodos de A até $\mathrm{D}$, não foram significativos. Assim, optou-se pelo uso do Método 1 para se obter as equações que relacionam a velocidade medida com $\mathrm{N}$ frutos e aquela em que se usa Teor de umidade (\% b.u.) 1 fruto para as outras variedades e graus de maturação.

A Figura 4 (A e B) apresenta, respectivamente, para frutos da variedade Catuaí e Timor, os valores estimados para a velocidade de um fruto, usando-se o Método 1 a partir das velocidades terminais de cinco frutos, V1 (5 grãos) e de dez frutos, V1 (10 grãos). Nessas figuras são mostradas, também, as equações ajustadas (regressão linear), com os respectivos coeficientes de determinação, para expressar a forma do funcional $\mathrm{f}_{1}$, Eq. (5). Observa-se que o pior ajuste foi para frutos "verde", da variedade Catuaí.

A Tabela 4 mostra, para cada teor de umidade, os resultados dos testes (Newman Keuls, ao nível de 5\% de probabilidade) provenientes da comparação entre as velocidades terminais médias para 1 fruto, determinadas experimentalmente, $\mathrm{V} 1$, e estimadas de amostras com cinco, V1(5) e dez frutos, V1(10).

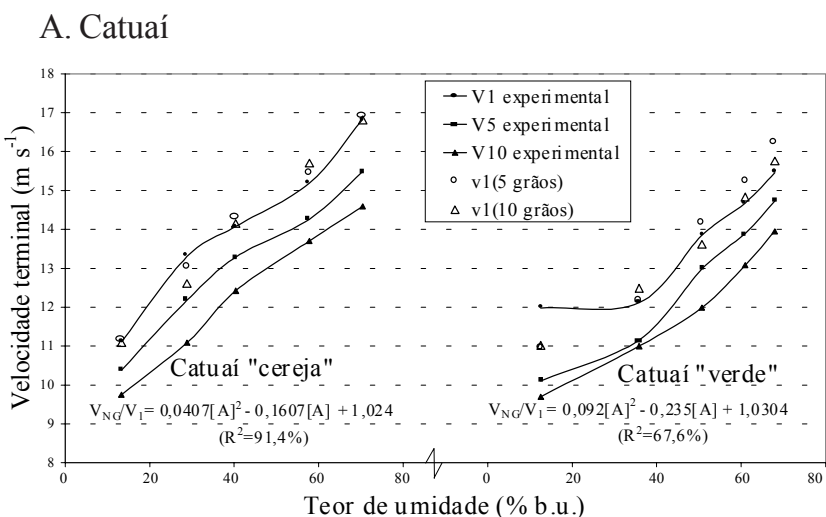

B. Timor

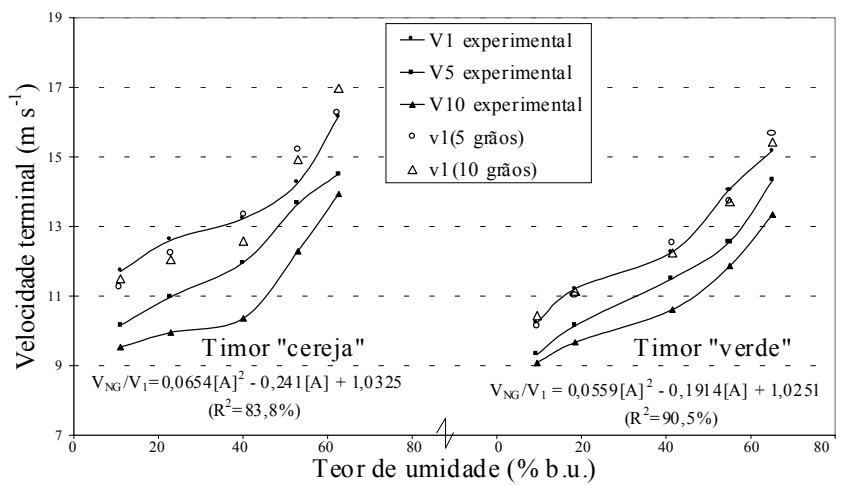

Figura 4. Velocidades terminais experimentais para amostras contendo um, cinco e dez frutos, da variedade (A) Catuaí e (B) Timor, e valores estimados para a velocidade de um fruto, pelo Método 1, a partir das velocidades de 5 e 10 frutos

Pode-se observar, na Tabela 4, que as melhores estimativas para os valores da velocidade terminal de um fruto foram obtidas para amostras da variedade Timor, contendo frutos "verde"; em segundo lugar, figuram as amostras da variedade Catuaí com frutos "cereja" e, neste caso, as velocidades para 1 fruto,

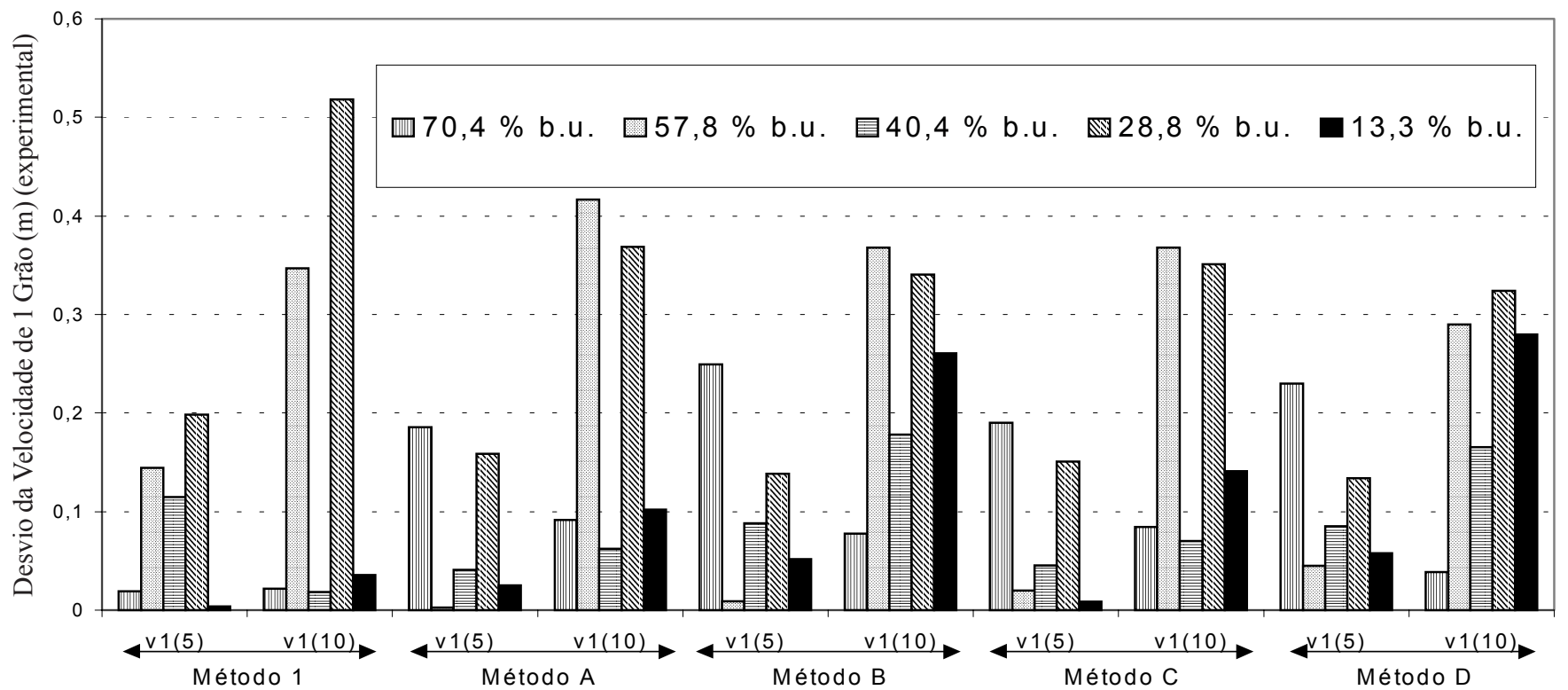

Método para Determinação de V1

Figura 3. Desvios nos valores das velocidades terminais estimados pelos diferentes métodos, para um fruto, ao serem usados os valores experimentais das velocidades medidas para cinco e dez frutos 
Tabela 4. Comparação entre as velocidades terminais médias de um fruto, determinadas experimentalmente e estimadas de amostras com diferentes números de frutos

\begin{tabular}{|c|c|c|c|c|c|c|c|}
\hline \multirow{2}{*}{ Variedade } & \multirow{2}{*}{ Grau de Maturação } & \multirow{2}{*}{ Condição de Comparação } & \multicolumn{5}{|c|}{ Teor de Umidade } \\
\hline & & & TU (1) & TU (2) & TU (3) & TU (4) & TU (5) \\
\hline \multirow[t]{5}{*}{ Catuaí } & "cereja" & $\mathrm{V} 1=\mathrm{V} 1(5)$ & $\mathrm{X}$ & $\mathrm{X}$ & $\mathrm{X}$ & $\mathrm{X}$ & $\mathrm{X}$ \\
\hline & & $\mathrm{V} 1=\mathrm{V} 1(10)$ & $\mathrm{X}$ & $(+3,42)^{*}$ & $\mathrm{X}$ & $(-5,23)$ & $\mathrm{X}$ \\
\hline & "verde" & $\mathrm{V} 1=\mathrm{V} 1(5)$ & $(+5,23)$ & $(+3,95)$ & $\mathrm{X}$ & $\mathrm{X}$ & $(-8,33)$ \\
\hline & & $\mathrm{V} 1=\mathrm{V} 1(10)$ & $\mathrm{X}$ & $\mathrm{X}$ & $\mathrm{X}$ & $\mathrm{X}$ & $(-8,03)$ \\
\hline & & $\mathrm{V} 1(5)=\mathrm{V} 1(10)$ & - & - & $\mathrm{X}$ & $\mathrm{X}$ & $\mathrm{X}$ \\
\hline \multirow{4}{*}{ Timor } & & $\mathrm{V} 1(5)=\mathrm{V} 1(10)$ & - & $\mathrm{X}$ & - & $\mathrm{X}$ & $\mathrm{X}$ \\
\hline & "verde" & $\mathrm{V} 1=\mathrm{V} 1(5)$ & $(+3,11)$ & $\mathrm{X}$ & $\mathrm{X}$ & $\mathrm{X}$ & $\mathrm{X}$ \\
\hline & & $\mathrm{V} 1=\mathrm{V} 1(10)$ & $\mathrm{X}$ & $\mathrm{X}$ & $\mathrm{X}$ & $\mathrm{X}$ & $\mathrm{X}$ \\
\hline & & $\mathrm{V} 1(5)=\mathrm{V} 1(10)$ & $\mathrm{X}$ & $\mathrm{X}$ & $\mathrm{X}$ & $\mathrm{X}$ & $\mathrm{X}$ \\
\hline
\end{tabular}

TU (1) - Maior teor de umidade e TU (5) - Menor teor de umidade

$\mathrm{X}$ - Ocorreu a condição

* Número entre parênteses $=$ percentual acima $(+)$ ou abaixo (-) em relação a V1 experimental

estimadas usando-se as amostras com 5 frutos e que foram sempre estatisticamente iguais. Nota-se, ainda, que nos casos em que ocorreram as melhores estimativas, as curvas para as velocidades terminais usando-se 1, 5 e 10 frutos (Figura 4A e B), apresentam comportamento aproximadamente similar.

Por fim e ainda na Tabela 4 encontram-se, também, para os casos em que foram detectadas diferenças significativas, valores percentuais indicando a superioridade ou inferioridade dos valores estimados em relação aos determinados experimentalmente para amostras contendo um fruto. Nota-se que os maiores valores percentuais são para frutos "verde", a uma umidade de $12,6 \%$ b.u., da variedade Catuaí. Observa-se, na Figura 4A, que o comportamento da velocidade terminal experimental para 1 fruto nas proximidades deste teor de umidade é bastante anormal.

Apesar da simplicidade das relações funcionais obtidas entre parâmetros adimensionais, usando-se análise dimensional e o teorema Pi de Buckingham, considera-se que elas forneceram estimativas razoáveis para a velocidade terminal de um fruto, em função das velocidades determinadas experimentalmente para amostras com um número maior de frutos. Eliminando-se a hipótese de problemas experimentais, os resultados poderiam, possivelmente, ser implementados se na análise dimensional fossem incluídos outros parâmetros relevantes ao processo, dentre os quais a queda de pressão, devido à obstrução do duto com frutos de café, parâmetros relacionados com a possível flutuação dos frutos em posições diferenciadas de acordo com o seu teor de umidade (quanto a diferentes rugosidades na casca) e parâmetros que expressem as forças de interação entre os frutos (contatos e impactos).

\section{CONCLUSÕES}

1. O teor de umidade dos frutos de café afeta significativamente os valores da velocidade terminal do produto. A velocidade terminal dos frutos diminui com a redução do teor de umidade do produto.

2. Antes do processo de secagem, o estádio de maturação dos frutos influencia nos valores da velocidade terminal do produto. Frutos “cereja" requerem, para flutuar, maiores fluxos de ar que os "verde".

3. A época de colheita de frutos da variedade Catuaí não afeta significativamente os valores da velocidade terminal do produto.

4. A variedade dos frutos (Catuaí e Timor) não afeta os valores da velocidade terminal do produto. Os desvios devidos à variedade encontram-se na mesma faixa dos experimentais.

5. O efeito do tamanho da amostra usada na determinação da velocidade terminal do produto é significativo. Valores de velocidades terminais determinados com amostras contendo 1,5 e 10 frutos, diferem significativamente.

6. Relações funcionais obtidas entre parâmetros adimensionais, usando-se análise dimensional e o teorema Pi de Buckingham, forneceram estimativas razoáveis para a velocidade terminal de um fruto, em função das velocidades determinadas experimentalmente para amostras com um número maior de frutos.

\section{LITERATURA CITADA}

Allen, C.A.W.; Watts, K.C. Properties of cowpeas (var Minica Beans). Journal of Agricultural Engineering Research, Silsoe, v.68, n.2, p.159-167. 1997.

Carman, K. Some physical properties of lentil seeds. Journal of Agricultural Engineering Research, Silsoe, v.63, n.2, p.8792. 1996.

Fox, R.W.; McDonald, A.T. Introdução à mecânica dos fluidos. LTC- Livros Técnicos e Científicos, 1998. 662p.

Gupta, R.K.; Das, S.K. Physical properties of sunflower seeds. Journal of Agricultural Engineering Research, Silsoe, v.66, n.1, p.1-8. 1997.

Mohsenin, N.N. Physical properties of plant and animal materials. New York: Gordon and Breach Science Publishers, 1978. 742p.

Nimkar, P.M.; Chattopadhyay, P.K. Some physical properties of green gram. Journal of Agricultural Engineering Research, Silsoe, v.80, n.2, p.183-189. 2001.

Singh, K.K.; Goswami, T.K. Physical properties of cumin seed. Journal of Agricultural Engineering Research, Silsoe, v.64, n.2, p. 93-98. 1996. 
Srivastava, A.K.; Goering, C.E. e Rohrbach, R.P. Engineering principles of agricultural machines. St. Joseph: American Society of Agricultural Engineers. 1993. 601p.

Tabak, S.; Wolf, D. Aerodynamics properties of cottonseeds. Journal of Agricultural Engineering Research, Silsoe, v.70, n.3, p.257-265. 1998.
Teixera, M.M. Limpeza de grãos. Viçosa: CENTREINAR, 1995. $31 \mathrm{p}$.

Uhl, J.B.; Lamp, B.J. Pneumatic separation of grain and straw mixtures. Transactions of the ASAE, St. Joseph, v.9, n.2, p.244-246, 1966. 